\title{
TülSt (transformable über interface for stardom)
}

\author{
Rui Pereira
}

\author{
4112 29th street apt.\#3 \\ Long Island City - New York \\ +1917340 2057 \\ rpp227@nyu.edu
}

\begin{abstract}
This project is an emotionally driven interface taking on our inner desires and fantasies of instantaneously becoming superstars ( and momentarily living under the skin of our idols) and on our instinctive imitation of the musical performers gestures as expressions of sounds.

TüISt is a multi-instrument interface based on a single object with minimal gesture input surfaces and various modes of use through different positioning and orientation towards the user's body. Multi-track gesture recording and playback/looping features also enable for multi-arrangement and composition by allowing the user to record and interact with his own performances in time. The objective was to develop an intuitive and playful interface for novices, an interface capable of providing explorative interactions in an enjoyable experience inspired by our significant mimicking gestures of 'real' musicians and our private ambitions of creating music.
\end{abstract}

\section{Keywords}

Nime, toy, motor-mimesis, gesture, multi-track, one-man-band, transformable.

\section{INTRODUCTION}

'...music does not express this or that particular and definite pleasure, this or that affliction, pain, sorrow, horror, gaiety, merriment, or peace of mind, but joy, pain, sorrow, horror, gaiety, merriment, peace of mind themselves, to a certain extent in the abstract, their essential nature, without any accessories, and so also without the motives for them.' [1]

Since the early days of humanity, along with the first artifacts made, musical instruments made of pieces of bone and animal skins could be found. Throughout the ages there is an undeniable and unquestionable assertion on the importance of music on societies and (more importantly) on individuals. Music has an omnipresence in the story of humanity, an essential part of our rites and ceremonies, celebrations and social affirmations. For hundreds of years we all knew how to sing and how to dance and did it as naturally as we talk, nevertheless, about five centuries ago a gap was created and kept expanding throughout time: music as a social manifest and lubricant produced and experienced by all the people, suddenly,

(C) The Author 2009.

Published by the British Computer Society started being played by some fortunate (qualified) ones only to be listened by others[2]. This breach is even more evident nowadays with the mythification of the performer by the media. Radio, TV (and ultimately the internet) didn't only replace the traditional value passing through storytelling but, also, the family gatherings around a musical instrument (lets not forget the role of the piano as one of the first home entertainment systems) or simply their voices and clapping hands. But, if this more intimate sharing of values, manifests and emotional expressions was put away; recorded music, ether and cable have brought us global experiencing and (sometimes) instantaneous access to media in cumbersome varieties and origins but also standards and conventions. The mass media market has also taken advantage of our need to live our emotions and fantasies through music. If on the one hand, music's pure form of emotions and abstraction allows us to recognize ourselves and our own feelings and states of mind; on the other hand, massmedia transformed the musician into a myth and hero of our times, modeling our dreams, desires and wishes to mirror our stage idols.

Even though the advent of electronic and digital technologies as brought a wide range of almost limitless possibilities in the creation of musical instruments, most of those were developed for musicians, involving long learning processes, complexity and a seriousness that many times prevents non-musicians from using them.

\section{IMITATION, GESTURES AND FANTASIES}

Imitation has an extremely important role in our learning process and social customization: we learn by imitating, by watching others actions and repeating them when faced with similar situations or context to those of the originally observed. Imitation and play are part of our integration and understanding of the world. Unfortunately, as we grow up and we start loosing our pursuits of play and our role playing and experimentation activities. Those gestures and body expressions that so much enhanced our imagination and boosted our beliefs in the reenacted fantasies are many times forgotten or, else, refrained to more private environments.

By mimicking the performer's gestures, children transpose themselves into the musicians body with the truly belief that its their (the children) actions that produced the sound and not the musician's. These re-enactment procedures and their important part on our perception and cognition processes besides demonstrating a strong personal and emotional involvement with the music also strongly imply that a mental re-play of sound-producing gestures would be part of making sense of sound.

In fact, according to recent research studies [3] [4] [5] [6], images of gestures seem to be efficient in evoking images of sounds: gestural imagery, mental simulations of gestures can 
trigger and/or enhance images of musical sounds. Another point refrained is a strong correlation between the activation of certain motor areas of the brain when listening or imagining sound. These studies also divided the types of gestures into two categories: excitatory and modulatory actions. Excitatory gestures refer to the sound-producing actions, short burst of energy, and the transfer of that energy into the body of an instrument. Modulatory actions are gestures produced with the intention of changing, modifying the resonant nature of the produced sound. These different gesture modes are associated with affective, emotive gestures which may potentially include all the movements and/or mental images of movements associated with more global sensations of the music.

Ultimately, it is this performance driven emotion and imitation, supported by our mirror-neurons and their physical transposition of the performer's actions and inner (body and mind) space into our own bodies that is intended to explored through the research and development of the project TüISt.

It is this project's objective the construction of an interface that uses our natural and subjective interpretation of the musician's gestures and uses these body expressions and gestures as the control of the interaction with the device: promoting a free performance of a musical instrument without any musical knowledge or ambition besides the emotion of mimicking our stage myths.

\section{INTERFACES REFERENCES}

The advent of digital technology and the especially the personal computer have, in many ways, reduced the gap between listeners and music makers: if the flexibility and power of modular software tools leveraged the audio manipulation and music creation possibilities to almost limitless of control, also its standardization, easy distribution and consequent widespread, together with the demystification of the digital technology have given us now more access than ever to knowledge, tools and chances of creating and manipulating musical sounds.

This massive access and use of digital music technology has also promoted standards and conventions, many times discarding the aspects of performance and emotional expressions in advantage of multi-functionality, multiparameterization and general use.

New instruments and musical interfaces, allied to novel models of interaction and performance are increasingly appearing and easing our (artistic) lives and needs, a good historical perspective on these instruments can be found in [7]: complex and complete systems for the 'virtuosos', but also, smart, instantaneous/direct interaction based instruments and toys for the 'dumb' and kid that lye in us.

\subsection{Theremin}

The invention of new musical instruments has not only been thrust by the search for new sounds and sonic explorations but also by the development of more expressive interfaces. The theremin (1917) aside from being an electronic music pioneer is still one of the most expressive and original musical instruments ever made. Its technological innovation and radical difference from traditional models in both sonic and performance areas (electronic) was enthusiastically embraced and the excitement with the futuristic instrument sometimes led to exaggerated assumptions:

"It is claimed that anyone who can hum or whistle a tune is able to produce whatever music he wishes from the Theremin. Little technical knowledge, practice or study is necessary in order to play it. . . " [8]

The theremin's gestural interface and non-existence of a resonant surface promoted wider gestures and more informal interactions but certainly did not imply any lack of precision or skills. A rather exquisite and rigorous interface, demands studied and extremely precise gestures, becoming the instrument of the lives of many musicians with classical background such as virtuosos Clara Rockmore or Pamelia Kurstin. Later on L.Theremin developed a theremin-cello continuing to feature his minimal approach to interfaces but as it's older brother this wasn't in fact an instrument for nonmusicians as most of the instruments developed afterwards in the wide range of possibilities provided by electronic and digital technologies. quickly welcomed in the more traditional classic music environments.

Even though there is a lack of materiality, no hitting, lucking or striking resonant objects) but instead a pair of antennas inviting to wider gestures or their possibility, the sensing technology used in the theremin (radio frequencies) reduces the length of the gestures to precise actions of proximity distance towards the antennas and a need of constant vigilance on full body movements, leveraging the needs of expertise in order to play this instrument.

\subsection{Yamaha Miburi}

On the course of this research several references [9] [10] were found on Yamaha's Miburi - an avant-garde fruit from Yamaha's design labs in the 90's. This wearable interface allows its users to control and play several virtual musical instruments through similar gestures used in the performance of their real versions. Miburi was launched in 1996 and later on a wireless version was released on the Japanese market only.

It is a modular interface built upon the networking of several wearable gadgets/controllers consisting of a suit (with flex sensors in 3 joints - wrist, elbow and shoulder), grip units for both hands ( 8 keys for triggering), foot sensor and a belt as a signal distribution unit and connection to the Synth and speakers unit. Its natural mappings of the gestural patterns and inherited virtual interaction spaces/locations (correspondent to the physical instrument spatial configuration) suggest a wider and more expressive interaction. The lowered resolution and approximate nature of its gestures with fixed spatial coordinates seems to be completed by an accompanying action/triggering on the handheld 'grip units' in order to achieve a higher precision in the control.

Miburi's sensors network seems to provide an effective gesture capture system allowing for different mapping functions to different instruments actions.

The available modes of gesture capture can also be redefined and configured by the user to its needs, providing along with its midi standard implementation a diverse range of uses and adaptation [10]. Nevertheless, this flexibility of configuration and programmability will demand expertise and technical knowledge but also more time and dedication.

In my regard, even though inspired by natural and spontaneous gestural expressions, its complex combination of actions and programmability so attractive and needed for experts precision needs can also become a become a barrier for novices use. 


\subsection{Guitar Hero}

Gaming and entertainment technology developers, after spending years exciting our brains and fulfilling our fantasies with high-action, high-powered machinery simulations seem now to have discovered a new niche for our emotional stimulation: on-stage superstars experience re-creations. This is without a doubt the case of GuitarHero [11] and RockBand [12], two games created in the recent years and which rapidly conquered the market with their very own game genre. Available for the most of gaming platforms in the market and featuring exclusive and specialized game controllers cloned from conventional musical instruments, these games are also supported by the emotional exploitation of our inner desires to (at least momentarily) become rock'n'roll stars, driving its players through controlled on-stage live action. Unfortunately the flow of interaction in the game is pretty much closed and stuck to the machine/computer commands, closing itself to more explorative interactions and creative impulses, not being much left for the player other than repeating the asked actions/orders in the right time and sequence. Its plastic controller and its discrete push-buttons featuring a characteristic cheap and cranky sounds when pressed (almost overlapping the game's tune and sound effects) refrains any possibility of expression still left.

But, as a game, and not a musical instrument, its objectives an priorities and not those of creative expression but those of challenge and dispute supported by the simulation of fantastic and uncommon experiences and environments with no consequences. In my perspective, the use of a game controller with such a close resemblance to the musical instrument performed in the virtual world of the game does in many ways help to augment this experience and its immersion.

\section{PROJECT DESCRIPTION}

TüISt is a new interface based on/ inspired by well known and established kinds of musical instruments and their performative tasks. Through its defined usable positions and modes there is an easy recognition and immediate association to the inspirational models and thus promoting cross references to their performance gestures and motor/musical imagery connections.

The objective was to create an easy to use, explorative interface with multiple personalities while resting on the same input surfaces and the ability to play these different instruments simultaneously. There is an intent to explore connections between musical and gestural imagery as a basis for its interactivity and to allow the user to perform with himself as a 'one man band'.

It's novelty comes from: multi-instrumentation without physical reconfiguration; similar gesture capture devices for different instruments modes; approximative and lower resolution gestures; independent multitrack recording and playback of gestures.

Its explorative interaction model promotes both experimentation and training, by minimizing its main features/gesture's capture devices. Thus, logical constraints limit to an also reduced set of accessible parameters, reducing the range of sound creation and manipulation possibilities. Nevertheless the multi-instrumentation capabilities allied with independent multiple tracks for gesture recording and playback should exponentially increase it's models of use, functionalities and creative output.

The experience is greatly fueled by the possibility of multi-layering of previously recorded gestures with currently happening gestures, allowing the user to interact with his own performance gestures/actions over time. This possibility of playing back, re-listening and playing along can promote an awareness of the musical or lack of musical qualities and/or attributes in each performance by their adaptability and ease of accompanying as well as more structural and compositional awareness.

TüISt does not smooth and enhance the results of the experience: it won't make the user's actions sound good or better. Therefore it is implied an eased learning curve on its manipulation. Nevertheless this process is eased by the arrangement and accompaniment features provided by the multi-track recording and playback possibilities. As a 'too smart' instrument[13] might have a shorter life-spam and quick lost of interest but is easily grasped and easily explored i tried to fight this effect by expanding its capabilities and multifunctionalities and also the affective/emotive background. Not only its multi-functionality and multi-instrumentation but also the multi-track features implemented are strong steps into prolonging its life-spam.

\subsection{Instruments}

Through the analysis of selected instruments with recognizable value in music creation and production and respective associated gestures, parallels were created in their performing actions and types of gestures involved in their performance. Triggering and modulating actions - reactions were analyzed and grouped in order to create matching points in three different instruments: guitar, stand-up bass and tabla or hand-drums.
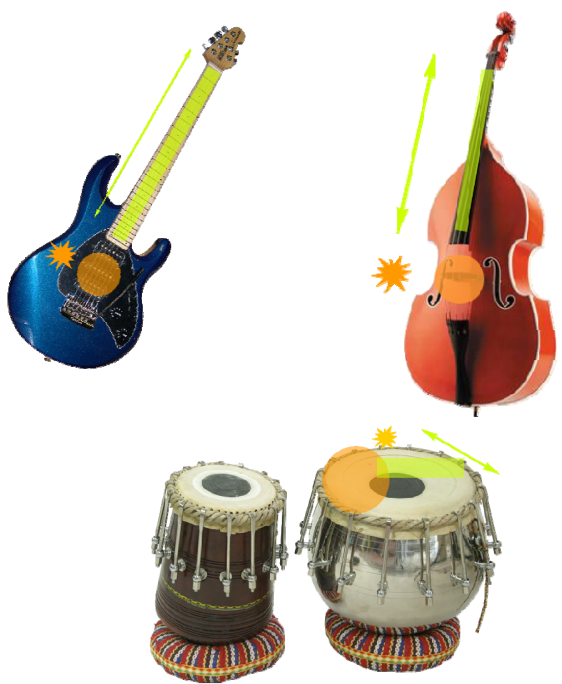

img. 1 - instruments modes of use (triggering and modulating sounds)

The possibility of choice and definition of one of the three different possible instruments is done through methods of position and orientation sensing. Each of the three instruments is associated with the natural position inferred by its inspirational model. The user can easily change the instrument mode by matching the interface orientation and position to the one of its inspirational models. By keeping that position for a brief period of time ( 2 seconds) the instrument gets locked into the correspondent specific mode. In this way there is a 
reinforcement of the identification of the instrument with the expected gestural interaction.

TüISt features a minimal interface by focusing on two main input surfaces: triggering (the sound-producing action) and modulating (the change or manipulation of the produced sound). This simplified interaction and analogy to the traditional instruments promotes obvious and logic interactions and instantaneous playability. By using the same mappings to the input surfaces in all instruments modes creating a coherent interaction. In all instruments modes there is a triggering action accompanied by a modulating - selective action to choose the tone of the produced sound. in both instruments this modulating action olis done by traveling along the body of the instrument in a gradient of tonality. Also, by maintaining similar mappings and functionalities throughout the different instrument modes promotes a faster adaptation and a quicker learning process.

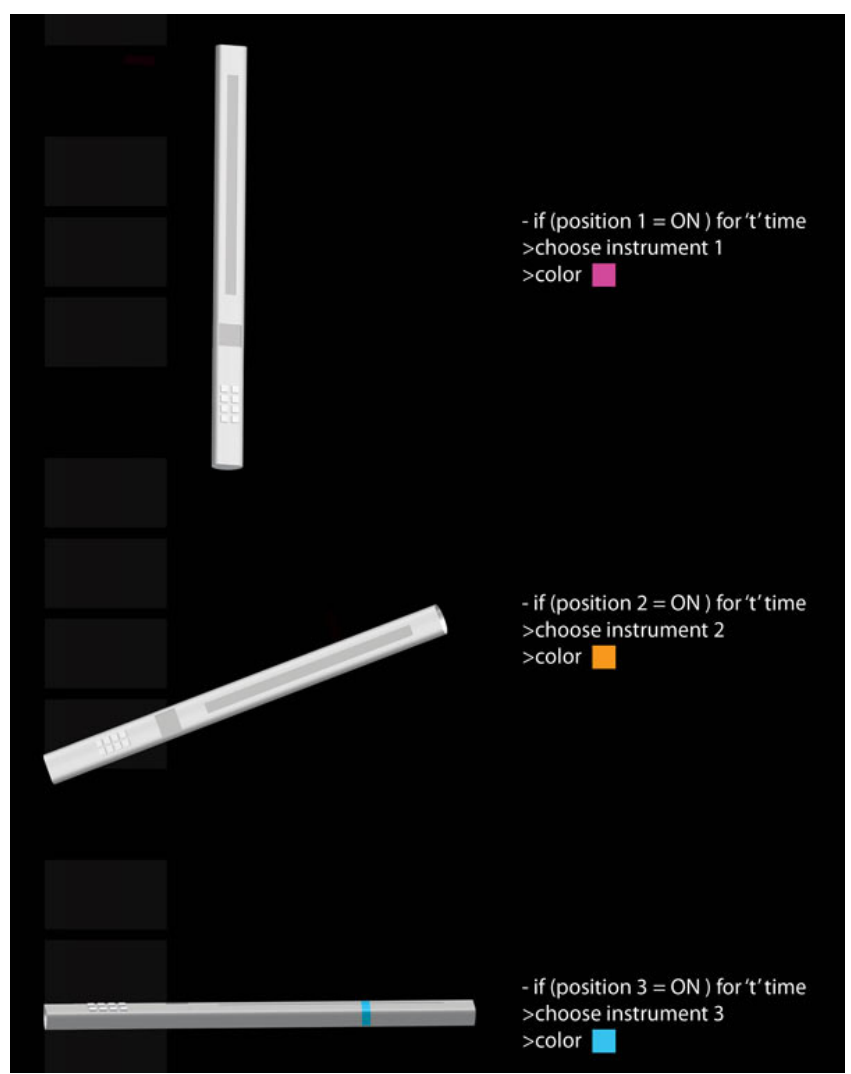

img. 2 - TüISt's instrument mode selection diagram

As the sound triggered by punctuated short bursts of energy has an inner/physic relation with its materiality by choosing a minimal structure fabricated with fiberglass it was intended a singular and synthesized \clone and blend of the three inspirational instruments DNA - reduced to their main features and input surfaces. The synthesized material look and feel was developed in order to promote the performance of its synth based sound engine. Each synthesized instrument tries to explore timbre similarities with the cloned reference.

In order to reduce the complexity and reduce the needs of precision and expertise to play the instrument some other actions were taken: reduced scale range accessibility to two or three octaves and notes are sequentially arranged from their lowest frequency in the extreme furthest away from the body rising to higher pitches/frequencies as we get closer to the body.
The mapping of the modulating sensor is done accordingly to actual physical models of string instruments following the conceptual model expectations.

\subsection{Multi-track recording and Playback}

Recording and playback modes permanently accessible. All their functions are quickly/immediately activated through one step interactions. TüISt features four configurable tracks each controlled by two independent specialized buttons. The first button (in order of closeness/proximity to the user's body) allows for track selection and activation functions. The second button is associated with the record and playback functions. Both buttons provide different color configurations and visual feedback related to their status and accessible action.

As an example of possible actions and correspondent visual feedback:

When starting the user defines the instrument by positioning in the desired instrument mode. As all tracks are empty there will be no color feedback. The instrument will be available for playing and experimenting. To start recording the performance the user has to simultaneously press both the desired track button and its associated control button. This coordinated action provides not only a security against accidental button presses and reinforces the change of mode (an evolution to a 2nd structural part of the interaction possibilities). As visual feedback for this action the track button light the color of the current instrument - promoting an eased future recognition of the nature of its contents. The control button activated recording state is signed by lighting red color. In order to stop recording a single press on the function button is needed. And even though the function button color will feature no light/color, the track color active state/color denotes the track is full/recording is over. By stopping the recording the user automatically access the track's playback functionalities. As feedback the track button will feature the recorded instrument's color ID as a visual reminder of it's nature and active state. Its correspondent function button will act as a toggle fro the track playback featuring green color lights or no color for Play - stop states respectively. As an extra feature during the tracks playback the user can restart the playback (cueing the playhead to the start of the timeline) by pressing the track button.

\section{SYSTEM DESCRIPTION}

The TüISt hardware system is composed of three main parts: position sensing, sensor data capture and function buttons access and feedback. The position system was developed using three tilt binary switches installed along the body of the instrument in different angles and orientations. As each of the sensors has its own specific location and angle inclination closely related to the defined instrument positions, by cross -comparing and combining their states possibilities it is possible to achieve confidant levels of certainty on the TüISt's position. The input sensors used for the sound producing and modulating actions were chosen by their main features and input possibilities according to the referred input gestures capture needs while paying special attention to their material and functional affordances and constraints in relation to the intended mappings. Both input surfaces feature analog soft touch sensing devices. As for the triggering gestures capture, a 2 " inch wide square shaped Force Sensing Resistor was used. This sensor provides a wide range and precise levels of pressure with a quite satisfactory sensitivity and response. 


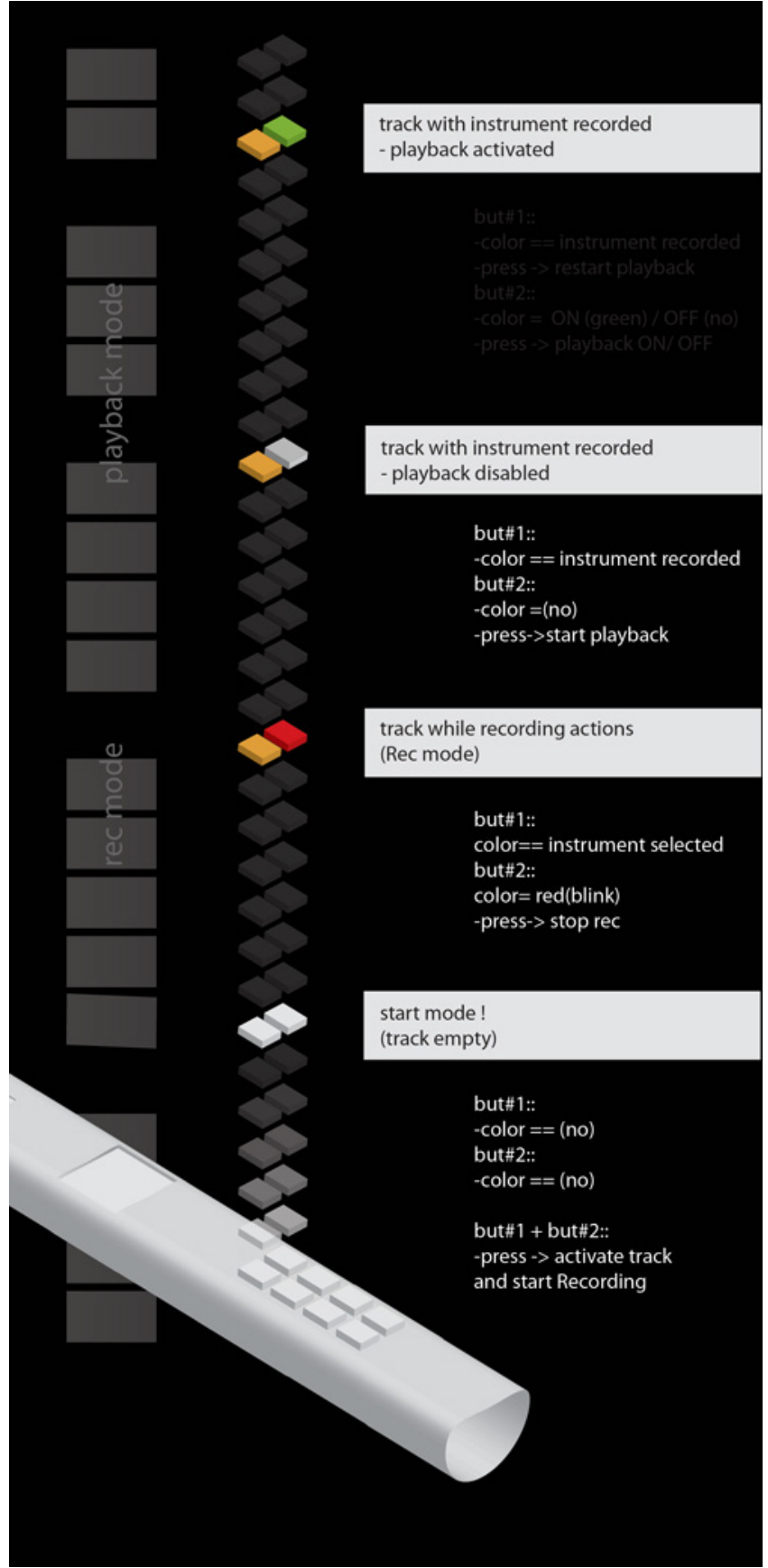

img. 3 - TüISt's button matrix diagram

In order to capture the modulatory gestures a touch linear pot is used. This linear position sensor allows for precise readings and location of touch along its extended $50 \mathrm{~cm}$ long surface. Even though it only detects the position but not the pressure of the touch, its long surface and precision combined with continuous touch sensing provide a trustful gesture capture. In order to enhance the touch experience and provide a physical feedback to the touch pressure and position, the sensor was laid in a bed of silicone soft rubber with a variable thickness related to the distance from the center of the instrument. Following the physics model of vibrating materials, the rubber's higher thickness and, therefore, more softness is located by the end of the instrument's body and starts diminishing and providing a less malleable / harder surface as we get closer to the triggering surface. My intention was to follow the physic model of a vibrating string and string instruments conceptual models.

The multi-track interface controls features a $2 * 4$ button matrix, each set of two aligned buttons is dedicated to one track. Its white translucent rubber pads allow for the use of embedded color Led's to provide visual feedback for each button.

All the track's functions, buttons states and visual feedback are exclusively controlled and managed by the micro-controller.

All the gesture sensor values, defined position and multi-track control functions are parsed and transmitted to Max/msp through serial connection protocol. The transmitted data packages include both analog sensors data, the current instrument defined position value and all the tracks status data wrapped in a customized Serial communication protocol.

All the input gesture sensors collected data and its manipulation is done at its higher resolution (0-1024) and only scaled to be converted to Midi data messages used to generate the synthesized sounds in virtual instruments software synthesizers.

\section{EXPERIENCE}

Users will be able to play and explore the TüISt Interface, experimenting its functionalities and their fantasies. A background projection featuring the user's contours and audio reactive graphical elements will allow for an augmented and more immersive experience. TüISt's visual component was built upon the realtime capture and transformation of the user's image. This feature was developed with the intention of creating not only a more immersive experience but also a complimentary hint to an on-stage performance simulation. The captured video image of the user is recreated in realtime in an aesthetic exploration inspired by colored light spots and laser beams reminiscent of grandiose live band performances.

Through methods of background removal and body shape contours finders a faithful image of the user is obtained and can be manipulated and transformed in reaction to sound. The transformation values are obtained through FFT audio analysis of the incoming the interface's sound signal.

If the TüISt interface multiplies its uses from different positions also the space provides different environments/modalities depending on the user's orientation. On-stage experience is provided by turning backs to the projection and facing the audience. For a more private experience the user can face the projection and experience the performance on his own.

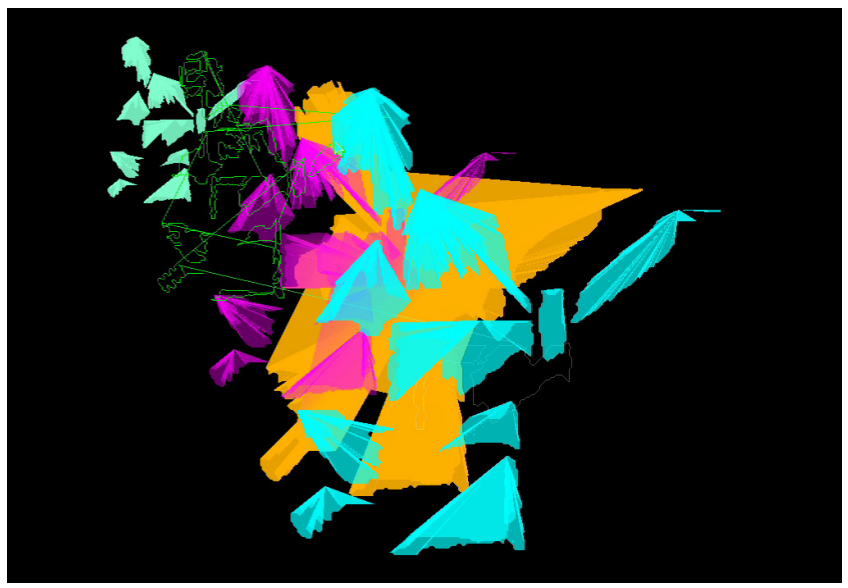




\section{REFERENCES}

[1] Levitin D. 2006, This is your brain on music: the science of an human obsession, Penguin Books,

[2] C.Cadoz 1999, Musique, geste, technologie. In H.Genevois and R. de Vivo, editors, Les nouveaux gestes de la musique, pages 47 -92. Editions Parenth'eses, Marseille.

[3] Godøy, R. I. 2003, Motor-mimetic music cognition. Leonardo 26(4): 317-19, MIT Press.

[4] Godøy, R.I. 1997, Knowledge in Music Theory by Shapes of Musical Objects and Sound-Producing Actions. In M. Leman (Ed.): Music, gestalt, and computing : studies in cognitive and systematic musicology. Berlin: Springer Verlag

[5] Godøy, R.I. 2004. Gestural Imagery in the Service of Musical Imagery. In A. Camurri \& G. Volpe (Eds.): Gesture-Based Communication in Human-Computer Interaction: 5th International Gesture Workshop, GW 2003, Genova, Italy, April 15-17, 2003, Selected Revised Papers, LNAI 2915. Berlin: Springer Verlag, pp. 55-62.
[6] Godøy, R. I., E. Haga, and A. R. Jensenius 2006. Playing 'air instruments': Mimicry of sound-producing gestures by novices and experts. In S. Gibet, N. Courty, and J.-F. Kamp (Eds.), Gesture in Human-Computer Interaction and Simulation: 6th International Gesture Workshop, GW 2005, Berder Island, France, May 18-20, 2005, Revised Selected Papers, Volume 3881/2006, pp. 256-267. Springer-Verlag GmbH.

[7] Paradiso, J. 1997. Electronic Music: New Ways to Play. IEEE Spectrum 12:18-30.

[8] 21st Century instruments

[9] Marrin, Teresa Anne 1996, Toward an Understanding of Musical Gesture: Mapping Expressive Intention with the Digital Baton, MIT

[10] Vickery, L. R. ,The Yamaha MIBURI MIDI jump suit as a controller for STEIM's Interactive Video software Image/ine. http://hub.guitarhero.com/

[11] http://www.rockband.com/

[12] Cook, P. 2001, Principles for Designing Computer Music Controllers, ACM CHI Workshop in New Interfaces for Musical Expression (NIME). 